\title{
Evolución de los intereses y la presencia de Estados Unidos en la triple frontera
}

\author{
Evolution of the interests and presence of \\ the United States at the triple frontier
}

\author{
José Antonio Peña-Ramos* \\ Joaquín Ferro-Rodríguez**
}

\begin{abstract}
RESUMEN
La Tri-Border Area ha suscitado gran interés este siglo en Estados Unidos. Este la ha considerado un posible foco de terrorismo yihadista, aunque — según muchas fuentes - Estados Unidos tiene otros intereses en el área, como el Acuífero Guaraní. El artículo aborda la verdadera presencia e intereses de Estados Unidos en dicha área, teniendo en cuenta tanto los proyectos relacionados con el agua, presentes en el hemisferio occidental, como la presencia del terrorismo en la región, con base parcial en los estudios del fiscal argentino Alberto Nisman. A partir de esos estudios, se realiza un análisis estructural prospectivo con la ayuda del software місмас, que indica un posible cambio de escenario.
\end{abstract}

Palabras clave: Estados Unidos - Tri-Border Area - Sistema Acuífero Guaraní - Seguridad - Terrorismo yihadista.

* Licenciado en Ciencias Políticas y de la Administración (2005, Primer Premio Nacional Fin de Carrera) y Sociología (2006, Premio a la Mejor Trayectoria Académica Andaluza, Academia de Ciencias Sociales y del Medio Ambiente de Andalucía) por la Universidad de Granada. Doctor en Ciencia Política (2009, Premio Extraordinario) por dicha Universidad. Desde 2010, Profesor de la Universidad Pablo de Olavide, desde $20 \mathrm{I2}$ como Profesor Contratado Doctor. japer@upo.es. Recibido: 27 de diciembre de 20I7. Aceptado: 24 de julio de 2018.

** Licenciado en Ciencias Políticas y de la Administración, y en Derecho (Universidad de Granada). Máster en Estudios Estratégicos y Seguridad Internacional en dicha Universidad, y Máster en Estudios Políticos Europeos en el Colegio de Europa (Brujas, Bélgica). Desde 2013, investigador en el Grupo de Estudios en Seguridad Internacional (GESI) de la Universidad de Granada. 


\section{ABSTRACT}

The Tri-Border Area has attracted great interest this century in the United States. It has been considered a possible source of jihadist terrorism, but according to many sources there are other US interests in the area, such as the Guarani Aquifer. The article discusses the real presence and US interests in the region, taking into account both the water projects that are currently being developed in the Western Hemisphere and presence of terrorism. Regarding the latter, this work is partially based on the investigations carried out by the Argentinian prosecutor Alberto Nisman. Building on this, this paper provides a prospective structural analysis with the assistance of МІсмAC software indicating a possible change of scenario.

Keywords: United States - Tri-Border Area - Guarani Aquifer System - Security - Jihadist terrorism. 


\section{INTRODUCCIÓN}

Durante la primera década del siglo xxi ha sido objeto de gran interés la Tri-Border Area (тва) de Brasil, Paraguay y Argentina (también conocida como Triple Frontier), punto geográfico donde convergen los tres Estados. Es una zona que, por diversas razones, concentra todo tipo de actividades ilícitas, de las cuales la que mayor temor y polémica ha suscitado en estos tres Estados y en Estados Unidos (EE. uu.) ha sido la eventual presencia de organizaciones terroristas yihadistas, principalmente a raíz de los atentados perpetrados contra objetivos israelíes en Buenos Aires, a principios de los años ' 90 .

Desde entonces, pero sobre todo tras el II-s, la твA ha sido objeto de una fuerte presión por parte de EE.Uu., que ha llegado a considerarla uno de los puntos más importantes desde donde organizaciones terroristas como AlQaeda o Hezbollah podían perpetrar nuevos atentados. Y aún cuando la presión estadounidense ha dado sus frutos en forma de colaboración antiterrorista con los tres Estados de la TBA, por la debilidad de las pruebas que han tratado de respaldar las acusaciones, estas han sido tachadas de falaces por numerosas fuentes latinoamericanas. Ellas han apuntado a que el discurso antiterrorista de EE.Uu. responde a otros intereses, sobre todo a la necesidad de aumentar su influencia en una de las zonas de recarga más importantes del Sistema Acuífero Guaraní (sAG) - considerada la mayor reserva subterránea de agua dulce del planetapara disponer de una alternativa a sus problemas de abastecimiento hídrico.

Este cruce de argumentos y acusaciones en torno al sag y a la TBa, que no ha permitido esclarecer satisfactoriamente la cuestión - como sucintamente apuntaron los autores en una versión reducida previa de la parte descriptiva de este artículo- ${ }^{1}$ y que durante la pasada década ha estado muy presente en las relaciones de EE.UU.con sus vecinos del sur, en la actualidad parece haber rebajado su importancia para los distintos actores.

Las preguntas que emergen, entonces, resultan evidentes: ¿qué hay de cierto detrás de los mutuos reproches y acusaciones? ¿Cuáles son los verdaderos intereses de EE.uU. en la zona? ¿Es posible que, tras una década de presión, Washington haya perdido el interés por la TBA? Y, de ser así, ¿a qué obedece?

El objetivo de este estudio es arrojar luces sobre el debate, siguiendo sus características y su evolución hasta la actualidad, desgranando los argumentos e intereses. Al hacerlo, lograremos una comprensión más fundada de la situación y seremos capaces de valorar cada

1 Véase Sitio Web de GESI: http://seguridadinternacional.es/?q=es/content/la-triple-frontera-el-acu $\% \mathrm{C}_{3} \%$ ADfero-guaran $\%$ $\mathrm{C}_{3} \% \mathrm{AD}$-y-los-intereses-de-estados-unidosen-la-regi\% $\mathrm{C}_{3} \% \mathrm{~B}_{3} \mathrm{n}$ 
factor adecuadamente, para descubrir qué hay de cierto en cada cuestión y, sobre todo, cuál es la dinámica actual y futura. El reto será, entonces, explicar cuáles son los intereses estadounidenses que verdaderamente han primado en la zona; cómo ha evolucionado el escenario regional e internacional, y cómo ello ha podido afectar y puede afectar, en el futuro, a dichos intereses y a la presencia de eE.uU. en la твa.

\section{Metodología}

Para alcanzar estos objetivos, se realizará un estudio de caso prospectivo, diacrónico e intensivo, que empleará la metodología del análisis estructural.

Szmolka y de Cueto definen los estudios de caso como "análisis intensivos sobre un solo objeto, sistema o país, que buscan proveer una detallada descripción sobre cómo las variables interactúan en una cuestión específica y en una instancia concreta, de cara a presentar generalizaciones cuya significación y validez vayan más allá del caso particular" (Szmolka y de Cueto, 20II: 215-216). Así, este estudio abordará un sistema, la твA, desde la óptica de un actor específico: EE.uU., y analizará de manera pormenorizada las variables que influyen en dicho sistema y que podrían afectar al comportamiento de dicho actor frente al mismo sistema.

Partiendo de autores como Guerrero (2006: I2), quien define los métodos prospectivos como "conjunto de análisis y estudios a realizar con el fin de explorar y configurar comportamientos y situaciones futuras", el estudio será prospectivo. Por tanto, no solo realizará una revisión de la evolución del sistema, sino que, teniendo en cuenta las tendencias pasadas y presentes, diagnosticará si efectivamente se está produciendo un cambio de escenario en la zona y cómo este afectará al comportamiento de los diversos actores en el futuro, centrándose en EE.UU.

El estudio también será diacrónico, precisamente por centrarse tanto en la evolución como en el presente y en el futuro del sistema, según Bartolini (I995: 44), para quien un estudio diacrónico consistía en que "los estados/ valores de las propiedades/dimensiones de los casos se modifican y 'varían' no solo de caso en caso, sino también para el mismo caso de un período temporal a otro". Finalmente, este estudio será intensivo, pues se han considerado "pocos casos y muchas propiedades o variables. Implica el conocimiento profundo de uno o pocos casos" (Bartolini, 1995: 44). En concreto, el sistema estudiado es solo uno, al tratarse de un estudio de caso, mientras que las variables son un total de 15 .

La hipótesis, cuya contrastación permitirá cumplir los objetivos enumerados, es la siguiente: "el cambio de escenario global propiciará una pérdida de interés y presencia de EE.uU. 
en la Tri-Border Area". La variable dependiente es el interés y la presencia de EE.uu. en la TBA, que dependerá de la evolución del resto, las variables independientes, las cuales se encuentran divididas entre 'actores estatales', en referencia a cada uno de los Estados considerados en el análisis, y 'características sistémicas', donde se incluyen los elementos que pueden haber llevado a EE.UU. a mostrar interés por dicha zona. Para realizar el cruce de variables y, posteriormente, comprobar la validez de la hipótesis, se empleará un tipo concreto de metodología prospectiva: la metodología del análisis estructural, con la que se pretende "describir un sistema con ayuda de una matriz que relaciona todos sus elementos constitutivos" (Godet, 2000: 68). Dicha matriz será elaborada con ayuda del software informático місмас, que nos ofrecerá una descripción de las variables más influyentes y de las más dependientes del sistema, y nos permitirá plasmar el funcionamiento del mismo en un gráfico que ilustrará su funcionamiento y facilitará su interpretación y compresión, así como el establecimiento de tendencias futuras (Godet, 2000: 68).

\section{La Triple Frontera}

El punto geográfico donde convergen las fronteras de Argentina, Paraguay y Brasil coincide con la desembocadura del río Iguazú en el río Paraná y es conocido como TBA, donde las ciudades de Foz do Iguaçu (Brasil), Ciudad del Este (Paraguay) y Puerto Iguazú (Argentina), forman un triángulo unido por el puente Tancredo Neves - entre Argentina y Brasil-y por el Puente de la Amistad - que une Brasil con Paraguay-. La superficie de la TBA abarca unos $2.500 \mathrm{kms}^{2}$, donde viven unas 700.000 personas que conforman una gran mezcla social y cultural, con la presencia de importantes colonias de ciudadanos alemanes, chinos, coreanos y árabes (Anzit, 2006). Particularmente, destaca una numerosa colonia de origen libanés que comenzó a formarse en la década de los ' 80 , a raíz de la llegada de inmigrantes que escapaban de la guerra civil libanesa y atraídos por las halagüeñas expectativas comerciales de la zona (Shaikhzadeh Vahdat, 20I3).

La твA se caracteriza, además, por su elevado nivel económico y comercial de tipo informal, favorecido por factores como la escasa presencia del Estado, la elevada afluencia turística y la porosidad fronteriza originada por la propia disposición geográfica de la zona. Estos elementos dificultan enormemente los intentos de fiscalización estatal y favorecen toda clase de actividades ilícitas, que han pasado a ser una seña de identidad de esa área. Así, 
la falsificación de documentos, la piratería de productos informáticos, el tráfico de drogas y de armas, la compraventa de automóviles robados, la trata de blancas o el lavado de dinero, son actividades habituales en la тва (Hudson, 2003: I0).

\section{Terrorismo}

Otra actividad que se han venido vinculando desde hace un tiempo con la твA es el terrorismo. Las primeras acusaciones sobre la presencia de grupos terroristas en la zona datan de comienzos de los años ' 90 , tras dos atentados en Buenos Aires que pudieron tener su origen en la тва: en 1992, un atentado suicida contra la embajada israelí en Argentina acabó con la vida de 29 personas, y en 1994 otro atentado contra la Asociación Mutual Israelita Argentina (AMIA) costó la vida a otras 85. La justicia argentina ha atribuido a Hezbollah la autoría de ambos atentados y ha apuntado a Irán como Estado cómplice e instigador, llegando a solicitar - sin éxito- la extradición de miembros de Hezbollah y de ciudadanos iraníes, entre ellos incluso el ex Presidente Rafsanjani y Soleimanpour, embajador iraní en Buenos Aires cuando se perpetraron los atentados. No obstante, los procesos judiciales que han tratado de esclarecer lo ocurrido se han visto enturbiados varias veces por obstrucción a la justicia y destrucción de pruebas, lo que ha causado el procesamiento, entre otros, del juez Galeano, que llevaba el procedimiento por el atentado contra la AmiA, y del ex Presidente argentino Carlos Menem, acusado de encubrir a un ciudadano de origen sirio (Caro, 20I2).

Sin embargo, fue a partir de los atentados del II-s cuando se intensificaron las acusaciones sobre la presencia de células dormidas de grupos yihadistas en la твA, acusaciones que se mantuvieron durante la primera década del siglo xxi. Después de que Bush lanzara su "guerra global contra el terrorismo", numerosos autores han apuntado directamente a la TBa como una de las zonas que más riesgo entrañaba para la seguridad de EE.UU. por acoger elementos terroristas yihadistas. No obstante, este tipo de afirmaciones no solo no han sido avaladas en ningún momento por hechos o pruebas irrefutables, sino que diversas fuentes oficiales en EE.uu. han admitido que no se han hallado pruebas concluyentes de que en la твA existan células activas o campos de entrenamiento pertenecientes a Al Qaeda, Hezbollah, Hamas u otro ${ }^{2}$. Sí está contrastada, en

2 Country Reports on Terrorism 2008. Recuperado el 06 de noviembre de 2017 de http://state.gov/documents/organization/I22599.pdf 
cambio, la presencia de movimientos, organizaciones y grupos islamistas, como la Yama'a al-Tabligh al-Da'wa, cuyas primeras visitas y viajes de misión a América datan de los años ' 70 del siglo $\mathrm{xx}$ - pero que pasaron inadvertidos por ser desconocidos y confundidos con otros-, hasta finales de los años ‘90, cuando comenzó a ser más visible, especialmente en Argentina y Brasil.

En realidad, la mayoría de los documentos disponibles en fuentes estadounidenses apunta más bien hacia la posible financiación del terrorismo, concretamente de Hezbollah, como la mayor amenaza en la región y la única sobre la que podrían encontrarse pruebas. Según estas fuentes, Hezbollah emplea las numerosas actividades ilícitas descritas, sobre todo el lavado de dinero, para financiarse. Las detenciones periódicas de ciudadanos de origen libanés en la zona, acusados de financiar a Hezbollah con este tipo de prácticas ilícitas, sobre todo en Ciudad del Este (Paraguay), han sido esgrimidas como argumento a favor de esta tesis. En este sentido, destaca la operación Barakat, en la que la policía paraguaya detuvo a Assad Barakat y lo acusó de financiar a Hezbollah mediante una trama de evasión fiscal llevada a cabo desde su red de comercio situada en un conocido centro comercial de Ciudad del Este. El Departamento del Tesoro de EE.uU. sostenía que Barakat era el tesorero de Hezbollah en la твA y proporcionaba a la milicia una cantidad aproximada de io millones de dólares anuales según el U.S. Naval War College (Levitt, 2007: I34-I5I). Sin embargo, fuentes latinoamericanas acusan de falta de rigor a las fuentes estadounidenses que mantienen este tipo de tesis, ya que las pruebas sobre las que estas se sustentan a menudo han sido consideradas débiles.

En cualquier caso, los tres Estados directamente afectados y EE.uU. han marcado la seguridad y la lucha contra el terrorismo en la тва como prioridad en sus agendas durante la pasada década. Desde 2002, los cuatro Estados han celebrado unas cumbres políticas conocidas como Grupo $3^{+1}$ para abordar la evolución de la тва y fomentar la mutua colaboración, intercambiar información, y mejorar la eficacia en la lucha contra el terrorismo y otras actividades ilícitas en el área triple fronteriza. Determinadas políticas, resultado de estas cumbres, han conllevado la intensificación de los controles estatales a todos los niveles, sobre todo en Brasil y Argentina. Controles que, sin embargo, no han agradado a los propios gobiernos, en la medida en que afectan negativamente sobre la actividad económica de la zona (Anzit, 2006: 6). En 2006, las presiones estadounidenses sobre el Grupo 3+I culminaron con la creación de un Centro Regional de Inteligencia, situado en Foz do Iguaçu, para vigilar permanentemente la твA (Gosman 2006). 


\section{SAG}

Bajo los territorios de Brasil, Argentina, Paraguay y Uruguay se extiende el Sistema Acuífero Guaraní (sAG). Se calcula su extensión en aproximadamente I.200.000 $\mathrm{kms}^{2}$, según el informe de Bruzzone, García y Ballester (2006). La mayor parte se sitúa bajo territorio brasileño; una superficie inferior, bajo suelo argentino; mientras que bajo $\mathrm{Pa}$ raguay y Uruguay hay una extensión más reducida. En la región abarcada por el SAG habitan unas I5 millones de personas, que emplean sus aguas para abastecimiento urbano, industrial y agrícola. Brasil es el Estado que más explota el acuífero, ya que unas 300 ciudades brasileñas utilizan sus recursos hídricos.

Según el mismo informe, el sAG contiene unos $50.000 \mathrm{kms}^{3}$ de agua, con una capacidad de recarga anual de $160-250 \mathrm{kms}^{3}$, lo que permitiría abastecer a 360 millones de personas con unos 300 litros de agua por habitante y día, si se explotaran solo 40 $\mathrm{kms}^{3}$ anuales. Además, el sAg cuenta con varios puntos de recarga, donde el agua se renueva, uno de los cuales —y de los más importantes_coincide precisamente con la твA.

Por su enorme potencial, el sAG ha sido objeto de gran atención. A comienzos del siglo xxi surgió entre los Estados que albergan el acuífero el interés de realizar un estudio de dos fases, para establecer, primero, las condiciones y características exactas del SAG, y segundo, una legislación conjunta que fomentara su explotación de manera eficiente y sostenible. Para ello se elaboró el "Proyecto para la Protección Ambiental y Desarrollo Sostenible del Sistema Acuífero Guaraní”, dotado con 27 millones de dólares cofinanciados por el Fondo Mundial para el Medio Ambiente (GEF), actuando el Banco Mundial (вм) como operador del presupuesto y la Organización de Estados Americanos (oEa) como ejecutora del proyecto.

Sin embargo, solo se culminó la primera fase, correspondiente al estudio científico, y el proyecto fue abandonado en 2009 tras las diferencias surgidas entre Uruguay y Argentina con motivo de la instalación por parte de Uruguay de dos plantas de pasta de celulosa sobre el río Uruguay, que separa ambos países (Fray Bentos, 2009). Ello impidió alcanzar algún acuerdo sobre un marco jurídico común respecto del SAG, un vacío legal que se trató de llenar en 20ro con un Acuerdo sobre el SAG firmado entre los cuatro Estados y auspiciado en el seno del Mercosur. Sin embargo, este acuerdo no entró en vigor porque no fue ratificado por Paraguay, Estado que argumentó que el contenido del Acuerdo vulneraba su soberanía sobre sus propios recursos, al establecer la negociación conjunta de las acciones que afectasen a dos o

3 Proyecto Sistema Acuífero Guaraní. Recuperado el 06 de noviembre de 2017 de http://sg-guarani.org/ 
más de los Estados que albergan el acuífero. No obstante, tras esta negativa podría ocultarse el resentimiento paraguayo con sus socios del Mercosur, sobre todo Uruguay, país que presionó para excluir a Paraguay de dicha asociación tras el juicio al ex presidente Fernando Lugo ${ }^{4}$. Así, hasta hoy han resultado infructuosos los intentos de elaborar una regulación regional o internacional sobre el SAG, cuya administración y explotación queda, por tanto, en manos de cada Estado, lo que puede originar un uso indiscriminado y poco eficiente del agua y la contaminación de la misma.

\section{Estados Unidos EN LA TBA}

Como se ha dicho, la твA ha despertado el interés de EE.uU. durante la mayor parte de la pasada década, sobre todo tras los atentados del II-s. Las alertas y acusaciones sobre la presencia de terrorismo yihadista en la zona, o sobre la financiación del mismo, se han venido reiterando asiduamente, aunque por lo general las pruebas aportadas han sido imprecisas. Ello ha motivado que desde fuentes latinoamericanas se haya argumentado que el discurso antiterrorista procedente de EE.UU. responde, en realidad, a otros objetivos. Concretamente, según diversas fuentes, mediante esta estrategia EE.UU. pretendería ganar influencia y presencia (diplomática y militar) en la твА para atender a estos intereses:

- Mantener estable y bajo control el flujo de hidrocarburos procedente de los Estados exportadores latinoamericanos, sobre todo Bolivia (Anzit, 2006: 9).

4 ABC Color (2012). "Rechazan el protocolo para manejo de Acuífero Guaraní," 3I de
- Reforzar su posición ante al conflicto colombiano, la actividad de las Fuerzas Armadas Revolucionarias de Colombia (FARC) y el narcotráfico (Anzit, 2006: 9).

- Contrarrestar la creciente doctrina anti-estadounidense instaurada en Latinoamérica, sobre todo tras la inclusión de la Venezuela de Hugo Chávez en el Mercosur; el aumento de las relaciones diplomáticas y comerciales de los Estados bolivarianos (y no bolivarianos) con Irán (Caro, 20I2), y los ambiciosos planes inversores chinos en la región (Ellis, 2013).

- Explotar el sag y conseguir una posición preferente respecto de posibles importaciones de agua para suplir sus carencias domésticas (Bruzzone, García y Ballester, 2006).

agosto. Recuperado el 06 de noviembre de 2017 de http://abc.com.py/edicion-impresa/ politica/rechazan-el-protocolo-para-manejo-de-acuifero-guarani-444985.html 
Este último es el que más ha llamado la atención y más protestas ha suscitado desde diversas fuentes latinoamericanas que han acusado a EE.UU. de pretender apropiarse de las aguas del sag, teniendo en cuenta que ello podría ayudarle a solucionar sus problemas internos de abastecimiento. Las propias comunidades islámicas de la твA han esgrimido este argumento para salir en defensa de las acusaciones de vinculación con grupos terroristas. En una entrevista a la ввс, el presidente de origen libanés del Centro Cultural Islámico de Foz do Iguaçu, Zaki Moussa, aseguraba que estas acusaciones obedecían al interés de EE.UU. de controlar las reservas de agua que se extienden bajo este lugar (Caro, 2012: 237). El propio informe de Bruzzone, García y Ballester (2006) apuntaba a la combinación de dos factores como principales elementos de riesgo para el SAG: el citado Proyecto de Sostenibilidad del SAG, coordinado por el BM, que podía otorgar a esta institución información privilegiada y facilitarle una eventual privatización de la explotación del acuífero y, posteriormente, otorgarla a multinacionales estadounidenses, sumado a las negociaciones sobre la creación de un Área de Libre Comercio de las Américas (ALCA) con la que EE.UU. pretendía crear un área de libre comercio a lo largo de todo el continente americano, para eliminar progresivamente los aranceles y, eventualmente, para importar libremente agua del sAG, y en la que el propio ALCA estableciera los mecanismos de resolución de controversias.

La escasez de agua a la que se enfrenta EE.UU. se ha convertido en un problema de importancia cada vez mayor en su agenda de seguridad (Biron, 20I2). Para paliarla, EE.uU. ha examinado diferentes opciones para adquirir y redistribuir agua mediante planes interiores y exteriores. Entre los primeros destaca el plan "Water 2025: Preventing Crises and Conflict in the West" de $2003^{5}$. Entre los segundos, tres son los más relevantes: el proyecto norteamericano (Canadá-EE.UU.) NAWAPA; el Plan Mesoamérica, que afecta a América Central, y el IIRSA, un proyecto de integración y aprovechamiento de los recursos para América del Sur, que incluye la construcción de infraestructuras de todo tipo y que podría afectar de forma directa o indirecta al SAG (Lasserre, 2005: I8-I9; Segrelles, 2010).

5 Water 2025: Preventing Crises and Conflict in the West. Recuperado el 06 de noviembre de 2017 de http://biodiversity.ca.gov/ Meetings/archive/watero3/water2025.pdf 


\section{Evolución}

Desde comienzos de la segunda década del siglo xxi, la información relacionada con el sAG y con la TBA ha disminuido, lo que podría significar que esta zona, objeto hasta hace pocos años de mutuos reproches y acusaciones procedentes de ambos hemisferios de América, haya comenzado a dejar de tener tanta importancia para los actores interesados. Ello podría obedecer a acontecimientos que se han producido en los últimos años, algunos de ellos en latitudes muy alejadas, pero que podrían haber modificado la percepción de EE.Uu. hacia la твA. Así pues, si consideramos ciertos los citados intereses estadounidenses en la zona, comprobamos que el escenario puede haber variado y afectado significativamente a los intereses y forma de presionar de EE.Uu. en la TBA. Comenzando por el primero de los intereses descritos, en los dos últimos años la Agencia Internacional de la Energía (AIE), en sendos informes anuales, ha anunciado que EE.UU. gozará de autosuficiencia energética hacia 2035 , en gran medida gracias a la explotación de hidrocarburos no convencionales a través del fracking (AIE, 20I3: I). Muchos análisis aseguran que este logro permitiría a EE.UU. centrarse en China y en la región de Asia-Pacífico y así disminuir su presión sobre Oriente
Medio ${ }^{6}$. Por ende y del mismo modo, ello podría suponer una relativa pérdida de presión sobre los hidrocarburos latinoamericanos.

En segundo lugar, el gobierno colombiano de Juan Manuel Santos, revalidado en los comicios parlamentarios y presidenciales de marzo y junio de 20I4, respectivamente, está negociando - al momento de redactarse este artículo- una paz con las farc que podría normalizar la participación política de la guerrilla. Además, históricamente este grupo se ha financiado con el narcotráfico, por lo que la resolución positiva del conflicto también afectaría al propio narcotráfico. Por ello, dicha resolución podría mermar los intereses de EE.uU. en la zona, y convertir en prescindibles determinados esfuerzos estadounidenses en ella. Respecto de la doctrina anti-estadounidense, desde Washington se había señalado el entendimiento entre Chávez y Mahmud Ahmadineyad como principal causa de preocupación, pues el carisma de ambos líderes en América Latina podía permitirles ganar cada vez más adeptos a su discurso anti-occidental y, específicamente, antiestadounidense. Sin embargo, la muerte del primero y la sustitución del segundo

6 Sustaining U.S. Global Leadership: Priorities for 2Ist Century Defense. Recuperado el 06 de noviembre de 2017 de http:// defense.gov/news/defense_strategic_guidance.pdf 
por el reformista Rohani — reelegido en 20I7- han debilitado este discurso y beneficiado a EE.UU. en esta lucha ideológico-discursiva, como demuestra el actual acercamiento entre Washington y Teherán, y el acuerdo nuclear entre Irán y el Grupo 5+I (los miembros permanentes del Consejo de Seguridad de la onu más Alemania). Ello, de paso, podría favorecer la imagen de EE.UU. entre los Estados latinoamericanos, pues al haberse reducido las sanciones contra Irán, a estos les resultará más fácil restablecer relaciones comerciales con el Estado persa.

En cuanto al SAG, los dos elementos que, según lo descrito, podrían facilitar su "saqueo" por parte de EE.uu., se encuentran actualmente paralizados. De un lado, ya se ha visto cómo las diferencias entre Argentina y Uruguay causaron el abandono del Proyecto de Sustentabilidad coordinado por el BM. Y, de otro lado, el ALCA no llegó a buen puerto y hoy parece inviable (Morán, 20II: I45), por lo que EE.UU. no podría acceder al agua del acuífero por esa vía si así lo pretendiese. Sin embargo, habrá que seguir prestando atención al desarrollo del IIRSA y a sus eventuales repercusiones sobre el acuífero; a la gestión que de este hagan los cuatro Estados que lo albergan, y a las presiones que sobre dichos Estados pudiera eventualmente ejercer EE.uU.con vista a la entrada de multinacionales en su explotación.
Para concluir, es preciso señalar que incluso el terrorismo, principal preocupación estadounidense reconocida en la zona, parece estar experimentando una evolución que lo desvincula parcialmente de la тва. Y es que en 2013 el fiscal general de la causa de la AMIA en Argentina, Alberto Nisman, presentó ante la justicia el "Informe Nisman" ", que advertía que Hezbollah ha trasladado su centro neurálgico en Latinoamérica a Venezuela, desde donde, según el documento, está dirigiendo dos redes de reclutamiento, financiación y expansión del terrorismo con apoyo irani ${ }^{8}$. Ese centro neurálgico radicaría concretamente en Isla Margarita, desde donde las redes se extenderían por toda América Latina (Noriega y Cárdenas, 20II), una de las cuales, según el Informe Nisman, estaría dirigida por Mohsen Rabbani, agregado cultural de la embajada iraní en Buenos Aires a principios de los años '90 y considerado por la justicia argentina como el cerebro de los atentados contra la embajada israelí y la AMIA? Bartolomé afirma que esto restaría a la твA importancia respecto del pasado (Bartolomé, 2013).

7 AMIA Case. Recuperado el 06 de noviembre de 2017 de http://defenddemocracy.org/ stuff/uploads/documents/summary_(3I pages).pdf

8 Ibid.

9 AMIA Case, op.cit., p. I. 


\section{ANÁLISIS}

Tras la descripción de las características de la TBA y el sAG, y a la vista del cruce de acusaciones que se ha venido vertiendo desde fuentes estadounidenses y latinoamericanas, es preciso realizar un estudio sosegado para arrojar luces sobre los intereses y la presencia de EE.Uu. en la zona. Para ello se realizará un análisis prospectivo de tipo estructural. Con la ayuda del software informático MICMAC, se obtendrá un mapa funcional donde queden representados los intereses estadounidenses en la zona, los diferentes actores que actúan en la misma y las relaciones de influencia y dependencia entre ellos. El objetivo es comprobar si los recientes acontecimientos expuestos suponen realmente un cambio de escenario que altere la percepción de EE.UU. sobre la тва. Primero definiremos, sobre la base de la información aportada hasta ahora, las variables a considerar:

\section{Variable dependiente:}

Interés y presencia de EE.UU. en la тва: los intereses de EE.UU. en la zona ya se han expuesto, por lo que los indicadores para seguir la evolución de dichos intereses y de la presencia estadounidense en la TBA son:

- Operaciones policiales y procesos judiciales relacionados con la zona.

- Interés del Gobierno o del Parlamento, así como provisión de fondos para operaciones especiales.
- Desplazamiento de tropas y operaciones bilaterales con los Estados implicados.

- Presión diplomática.

Variables independientes:

\section{Actores estatales:}

- Brasil: mayor potencia regional latinoamericana con aspiraciones de potencia global. Con gran apacidad económica y militar, pero acentuadas desigualdades sociales que fomentan violencia, crimen organizado y narcotráfico. Yacimientos petrolíferos recién descubiertos en manos de la compañía estatal Petrobras. Se erige como moderador y defensor de la democracia en la diplomacia regional. Mantiene relaciones estables con EE.uU., aunque propensas a tensarse por rivalidades de liderazgo. Tuvi una posición inicial ambigua sobre el ALCA, pero finalmente contraria, pues el acuerdo podía limitar su influencia regional (Morán, 20II: I45-I48). Según algunas fuentes procedentes de EE.UU., la presencia de Hezbollah parece estar consolidándose no solo en la твА, sino que en América Latina en general, gracias a la red de Rabbani (Noriega, 20I2: 2). No obstante, es el Estado que más controles efectúa en la твA para combatir el narcotráfico y el contrabando entre narcos brasileños y las farc (Anzit, 2006: 6). 
En las elecciones presidenciales de octubre de 20I4, la Presidenta Rousseff fue la vencedora, y en 2016 le sucedió Michel Temer. Ha acogido el Mundial de Fútbol de 2014 y los Juegos Olímpicos de 20I6, retos muy importantes para la seguridad.

- Paraguay: Ciudad del Este es la más insegura de las que conforman la TBA, donde menos presencia estatal existe y la más conflictiva (Hudson, 2003: II). No obstante, no es la única del entorno que es escenario de violencia y crimen organizado, pues en los últimos años la cercana ciudad paraguaya de Saltos de Guairá ha atraído estas actividades delictivas ${ }^{10}$. La asociación entre narcos brasileños y las FARC parece tener en Paraguay una ruta segura de traslado de mercancías (Anzit, 2006). EE.UU. y Paraguay tienen acuerdos militares de colaboración, y existe una base estadounidense en Mariscal Estigarribia (Sánchez de Rojas, 20II: I24).

- Argentina: sufre una crisis económica caracterizada por la inflación y la deuda que a menudo provoca graves crisis sociales. Importantes comunidades árabes y judías, aunque en Puerto Iguazú no son tan representativas. Dicha ciudad trifronteriza es la menos conflictiva de las tres, pero adolece de los mismos problemas económicos y sociales que toda

10 ABC Color. (20II). "Criminalidad se instala en Salto del Guairá, II de mayo. Recuperado el 06 de noviembre de 2017 de http:// abc.com.py/articulos/criminalidad-se-instala-en-salto-del-guaira-256318.html
Argentina y que sus dos ciudades vecinas, y destaca por el tráfico ilícito de documentos y vehículos (Hudson, 2003: 52, 55). La preocupación argentina por la твA está estrechamente vinculada a los atentados contra la embajada israelí y la AmiA. Hay que recordar que el Informe Nisman de 20I3 alerta sobre dos posibles tramas de propagación de Hezbollah apoyadas por Irán en Latinoamérica ${ }^{11}$. No obstante, el mismo 2013 Argentina e Irán firmaron un acuerdo de colaboración para resolver el caso del atentado contra la AmiA, aunque no parece estar dando los resultados esperados (Dinatale, 20I4).

- Uruguay: determinadas fuentes aseguran que el conflicto sobre el río Uruguay que mantuvo con Argentina fue la causa de que el Proyecto del sag respaldado por el BM no prosperase (Fray Bentos, 2009). Recientemente ha legalizado el consumo y la venta de marihuana bajo control estatal, vía monopolio, para paliar los efectos del narcotráfico, lo que puede tener consecuencias para toda América Latina.

- Venezuela: sumida en una profunda crisis económica, social y política. La buena relación existente entre los ex presidentes Chávez y Ahmadineyad ha preocupado a EE.UU. en los últimos tiempos. El mencionado Informe Nisman señala a Venezuela ${ }^{12}$, y concretamente a Isla Margarita

11 AMIA Case, op. cit.

12 Ibid. 
como el nuevo centro de organización terrorista de Hezbollah (Noriega y Cárdenas, 20II: 3). En EE.uU. se insistía en la permisividad del Gobierno de Chávez con el contrabando de armas y drogas entre Hezbollah y las FARC, y en que dicha red implicaría a altos cargos del propio Gobierno chavista (Noriega, 20I2: 4). Otro motivo de preocupación estadounidense era la inclusión de Venezuela en Mercosur, que Paraguay veía con recelo, pero al normalizarse las relaciones entre ambos Estados, su integración queda asegurada. Maduro carece del liderazgo de Chávez. La crisis de inflación y abastecimiento, así como el desfase tecnológico del país, le impiden gozar de la independencia de Chávez y le obliga a tener más en cuenta a una oposición ahora más unida y mejor posicionada ${ }^{13}$.

- Colombia: el narcotráfico y el crimen organizado en América Latina han estado estrechamente vinculados a las farc. Las rutas del contrabando han ido evolucionando e implicando cada vez a más Estados. Sobre todo se ha apuntado a Venezuela, Brasil y Paraguay. EE.uU. ya mostró su implicación en el problema, desarrollando el Plan Colombia, de 1999. El Gobierno de Santos se encuentra en negociaciones con

13 Stratfor. (2014). Venezuela's President reaches out to the opposition. Recuperado el 06 de noviembre de 2017 de http://stratfor. com/sample/analysis/venezuelas-presidentreaches-out-opposition las FARC para disolver a la guerrilla e integrarla en la política convencional. La resolución del conflicto afectaría claramente al narcotráfico en América Latina.

- Irán: sus conexiones con los Estados de América Latina han experimentado altibajos desde los dos atentados en Argentina. Sus relaciones comerciales se han visto afectadas por la dinámica internacional, fundamentalmente por las sanciones al régimen de los Ayatolas por parte de la comunidad internacional y las presiones estadounidenses a sus vecinos del sur para tratar de aislar a dicho régimen (Caro, 20I2). Sin embargo, durante el Gobierno de Ahmadineyad, Irán estrechó lazos con varios Estados, sobre todo Venezuela y sus aliados bolivarianos. El Informe Nisman señala la conexión terrorista de Irán con Hezbollah en América Latina ${ }^{14}$. No obstante, en 20I3 Irán y Argentina firmaron un acuerdo de cooperación para la resolución del atentado contra la AMIA, aunque no parece estar dando los resultados esperados (Dinatale, 20I4). Por otra parte, el acuerdo nuclear de finales de 2013 entre Irán y el Grupo $5^{+I}$ parece indicar un cambio en la visión estadounidense sobre Oriente Medio y un reequilibrio de fuerzas en dicha región (Friedman, 2013), que podría tener consecuencias para Hezbollah en América Latina.

14 AMIA Case, op. cit. 
- China: ha aumentado sus inversiones e intercambios comerciales en toda Latinoamérica, en el marco de una política de influencia expansiva por medio de los negocios, siguiendo una dinámica "sur-sur" que preocupa a Washington. Un claro ejemplo es el acceso de empresas chinas a concesiones en toda América Latina, para proveer componentes y participar en la construcción de infraestructuras relacionadas con las energías renovables, en las que China es líder mundial (Ellis, 20I3). Es el segundo socio comercial de Latinoamérica, donde sus proyectos inversores ascienden a 26.000 millones de dólares.

A continuación definiremos las características sistémicas:

- Sistema Acuífero Guaraní (SAG): las características, explotación e intentos de regulación internacional del sag ya se han expuesto. Hay que recordar la posible ratificación del acuerdo multilateral sobre el acuífero, ahora que las relaciones entre Mercosur y Paraguay están normalizadas. Estadounidenses adinerados han adquirido terrenos situados sobre el sAG, sobre todo en Argentina, para destinarlas, en teoría, a la protección medioambiental ${ }^{15}$. Destaca el proyecto IIRSA, que podría afectar

15 Martínez, Mausi (dir). (2004). Sed. Invasión gota a gota [Documental]. al sAG en alguno de sus puntos (Lasserre, 2005; Segrelles, 2010).

- Autosuficiencia energética de EE.UU.: la revolución de los hidrocarburos no convencionales podría permitir a EE.uU. alcanzar la autosuficiencia energética. Para 2035, EE.UU. podría cubrir todas sus necesidades energéticas con recursos propios, $\mathrm{e}$ iría reduciendo, paulatinamente, sus importaciones hasta el mínimo, incluidas las procedentes de América Latina. Ello supondría una reorientación del comercio energético mundial hacia Asia-Pacífico, que se convertiría en el mayor demandante mundial de hidrocarburos y el principal cliente de América Latina (AIE, 20I3: I).

- Geoestrategia estadounidense: la mencionada autosuficiencia energética estadounidense y el meteórico ascenso chino han propiciado que los intereses geoestratégicos de EE.UU. vayan desplazándose desde Oriente Medio hacia Asia-Pacífico ${ }^{16}$. EE.uU. trata de crear un sistema de pesos y contrapesos en Oriente Medio que garantizaría la estabilidad de esa región y le permitiría aliviar esfuerzos en ella para dirigirlos a contrarrestar el auge de China. Aquí se enmarcan acontecimientos como el acuerdo nuclear firmado con Irán o la evolución del conflicto sirio, que determinará el grado de interés de EE.uU. en Oriente Medio y en las áreas de influencia de los actores implicados

16 Sustaining U.S. Global Leadership, op. cit. 
—incluido Hezbollah—, en este caso la твA y en Latinoamérica en general. En la pasada década, la importancia de Latinoamérica para la seguridad estadounidense ha disminuido ante el ascenso de Asia-Pacífico, ya que en Latinoamérica, EE.UU. se ha centrado en el terrorismo, la delincuencia transnacional y la migración ilegal, y se ha basado en el "softpower" desde la llegada al poder de Obama, quien ha priorizado América Central sobre el Cono Sur (Sánchez de Rojas, 20II: I07-IIO).

- Situación del agua dulce en EE.UU.: en los próximos años, EE.UU. se enfrenta a una escasez de agua provocada por sobreexplotación de ríos, lagos y acuíferos; contaminación de los mismos; períodos de sequía que habitualmente afectan a ciertas zonas; desfase tecnológico de sus instalaciones hídricas, y crecimiento de la demanda por el incremento demográfico y urbanístico ${ }^{17}$. Tendrá que realizar reformas y elaborar planes de acción interior y exterior que le procuren un acceso al agua según sus necesidades y objetivos. Entre los planes exteriores hay que considerar el nawapa, el Plan Mesoamérica y, sobre todo, el IIRSA, que afecta directamente a Sudamérica y al SAG (Lasserre, 2005; Segrelles, 20I0). Respecto del sAG, el análisis tendrá en cuenta el proceso de evolución descrito y las opciones reales de EE.UU.

17 Water 2025, op. cit. de extraer su agua y transportarla a Norteamérica.

- Terrorismo: la evolución y estado actual del terrorismo en América Latina y la твA ya han sido expuestos. En esta variable se tendrán en cuenta principalmente la amenaza de Hezbollah en la тва y las consecuencias para esta organización de acontecimientos como la investigación de los atentados contra la AMIA y la embajada israelí en Buenos Aires, y la evolución de Irán.

- Narcotráfico: muy extendido en América Latina, afecta directamente a la твA. Esta variable tendrá en cuenta la evolución y estado actual del narcotráfico, los Estados y zonas de origen del tráfico de drogas, las rutas y estrategias más frecuentes, y las medidas de los distintos gobiernos para combatirlos. La твA ha sido tradicionalmente una zona propicia para todo tipo de contrabando, pero existen otras rutas a considerar, por las que transita mayor volumen de droga y que atraen mayores esfuerzos por parte de las fuerzas y cuerpos de seguridad (Ameripol, 2013).

Definidas las variables, a continuación y con la ayuda de micmac, las relacionaremos entre sí con el fin de comprobar si existe influencia directa entre ellas y, de ser así, cuál es la intensidad de dicha influencia. Para ello se otorgará un valor de 0 a 3 a cada relación, siendo $\circ$ una influencia directa 
inexistente y 3 la influencia máxima que la variable estudiada puede ejercer sobre aquella con la que se cruza. Se otorgará el valor "P" cuando exista una influencia directa potencial entre dos variables (Godet, 2000: 70). La Tabla I muestra los resultados:

TABLA I

Matriz de datos

\begin{tabular}{|c|c|c|c|c|c|c|c|c|c|c|c|c|c|c|c|}
\hline & 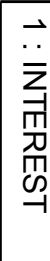 & 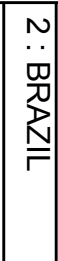 & 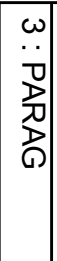 & 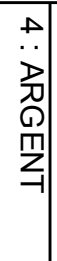 & 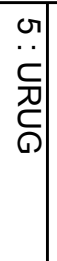 & & $\begin{array}{l}v \\
\dddot{\nu} \\
0 \\
\text { ๓ }\end{array}$ & $\begin{array}{l}\infty \\
\ddot{0} \\
\bar{p} \\
\bar{z}\end{array}$ & $\begin{array}{l}0 \\
\ddot{0} \\
\stackrel{\rho}{1} \\
\bar{z} \\
\end{array}$ & $\begin{array}{l}\overrightarrow{0} \\
\ddot{Q} \\
\stackrel{D}{ } \\
\mathrm{C}\end{array}$ & $\begin{array}{l}\overrightarrow{\mathrm{A}} \\
\mathrm{m} \\
\mathrm{m} \\
\mathrm{m} \\
\mathrm{d} \\
\mathrm{Q} \\
\prec\end{array}$ & 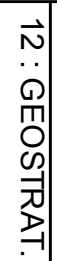 & 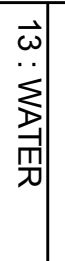 & 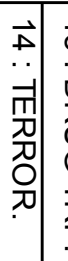 & 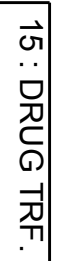 \\
\hline $1:$ INTEREST & 0 & 1 & 2 & 2 & 0 & $P$ & $\mathrm{P}$ & 1 & 0 & 2 & 0 & 1 & $P$ & \begin{tabular}{l|l}
3 & 2 \\
2
\end{tabular} & 2 \\
\hline 2:BRAZIL & 3 & 0 & 3 & 3 & 1 & 2 & 1 & 1 & 1 & 3 & 1 & 1 & 1 & \begin{tabular}{l|l}
3 & 3 \\
\end{tabular} & 3 \\
\hline 3: PARAG & 3 & 3 & 0 & 2 & 1 & 2 & 1 & 1 & 1 & 3 & 0 & 1 & 1 & \begin{tabular}{l|l}
3 & 3 \\
3
\end{tabular} & 3 \\
\hline $4:$ ARGENT & 3 & 2 & 2 & 0 & 2 & 2 & 0 & 2 & 1 & 3 & 1 & $\mathrm{P}$ & 1 & \begin{tabular}{l|l}
3 & 1 \\
\end{tabular} & 0 \\
\hline $5:$ URUG & $\underline{0}$ & $\mathrm{P}$ & $\mathrm{P}$ & 1 & 0 & $P$ & $\mathrm{P}$ & 0 & 1 & 3 & 0 & $\mathrm{P}$ & 1 & \begin{tabular}{l|l}
0 & 1 \\
\end{tabular} & 0 \\
\hline $6:$ VENEZ & 1 & 2 & 2 & 2 & 0 & 0 & 2 & 3 & 2 & 0 & 1 & 2 & 1 & \begin{tabular}{l|l}
2 & 2 \\
2
\end{tabular} & 2 \\
\hline $7: \mathrm{COLB}$ & 1 & 2 & 2 & 0 & 0 & 2 & 0 & $\mathrm{P}$ & 1 & 0 & 0 & 1 & 1 & $2 \mid 3$ & 3 \\
\hline 8: IRAN & $\bar{P}$ & 2 & 1 & 2 & 1 & 3 & 1 & 0 & 0 & 0 & 1 & 3 & 0 & \begin{tabular}{l|l}
3 & $F$ \\
\end{tabular} & $\mathrm{P}$ \\
\hline 9:CHINA & $\overline{1}$ & 1 & 1 & 1 & 1 & 2 & 1 & $P$ & 0 & $\mathrm{P}$ & 1 & 3 & \begin{tabular}{l|l}
$P$ & \\
\end{tabular} & \begin{tabular}{l|l}
0 & 0 \\
0
\end{tabular} & 0 \\
\hline $10:$ GAS & 2 & 2 & 2 & 2 & 2 & 0 & 0 & 0 & 0 & 0 & 0 & 0 & \begin{tabular}{l|l}
$P$ & \\
\end{tabular} & \begin{tabular}{l|l}
0 & 0 \\
\end{tabular} & 0 \\
\hline 11: ENERG & 2 & 1 & 0 & 1 & 0 & 1 & 1 & 2 & 2 & 0 & 0 & 3 & $\mathrm{P}$ & \begin{tabular}{l|l}
0 & \\
\end{tabular} & 0 \\
\hline $12: \mathrm{G}$ & 5 & 2 & 2 & $\mathrm{P}$ & $\mathrm{P}$ & 2 & 2 & 3 & 3 & 0 & 3 & 0 & $P$ & 3 & 3 \\
\hline 13: WATER & 2 & 1 & 1 & 1 & 1 & 1 & 1 & 0 & 0 & 2 & $\mathrm{P}$ & $\mathrm{P}$ & 0 & \begin{tabular}{l|l}
0 \\
0
\end{tabular} & 0 \\
\hline $14:$ TERROR. & 3 & 3 & 3 & 3 & 1 & 3 & 3 & 3 & 0 & 0 & 0 & 3 & 0 & $\begin{array}{lll}0 & 3 \\
\end{array}$ & 3 \\
\hline 15: DRUG TRF & 2 & 3 & 3 & $\mathrm{P}$ & $\mathrm{P}$ & 3 & 3 & 0 & 0 & 0 & 0 & 2 & 0 & \begin{tabular}{l|l}
3 & $(1$ \\
\end{tabular} & 0 \\
\hline
\end{tabular}

Fuente: elaboración propia con мIсмAC

Una vez otorgados los valores a cada relación, el programa muestra una clasificación de las variables más influyentes y dependientes del sistema, así como queda de manifiesto en el Mapa Gráfico I, que sitúa cada variable a lo largo de un eje estratégico en atención a su influencia y dependencia. Para este sistema, el mapa es el siguiente: 
MAPA I

\section{Influencia directa/dependencia}

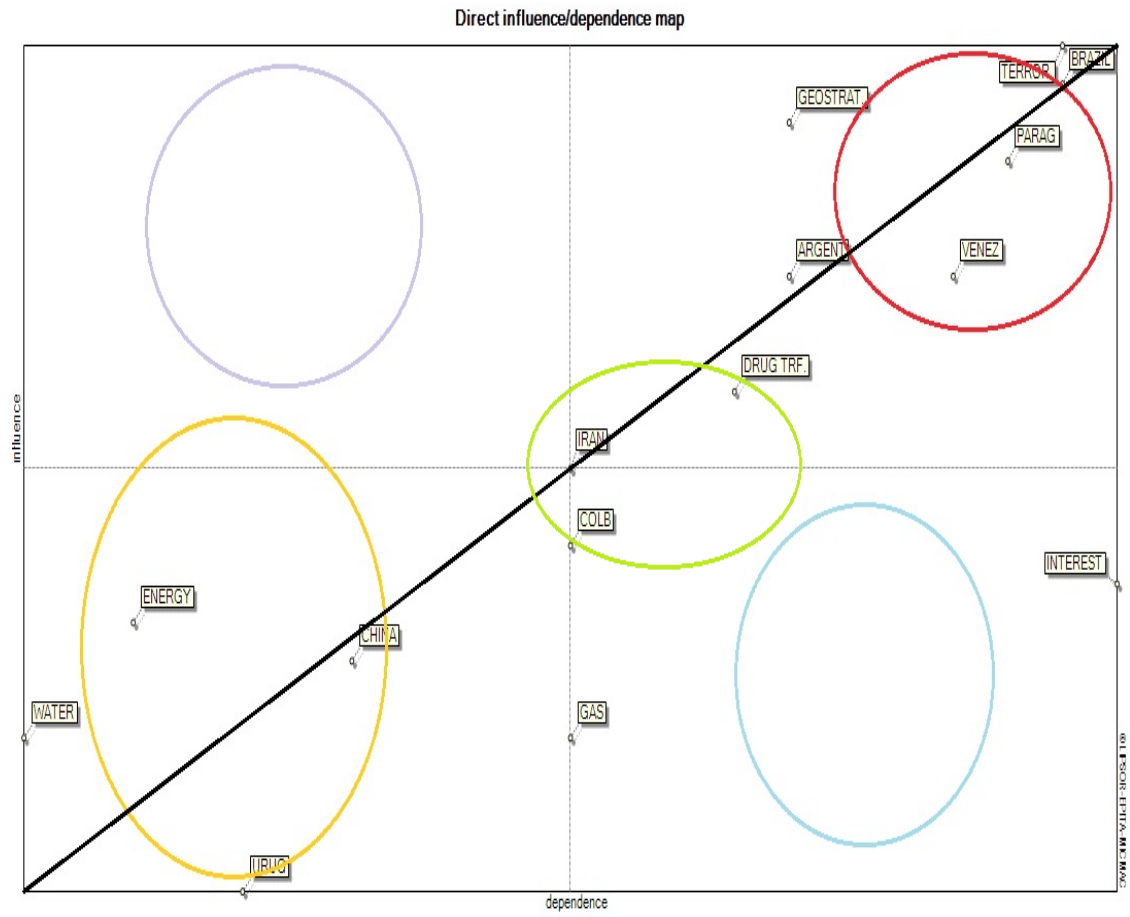

Clasificación de las variables según su posición

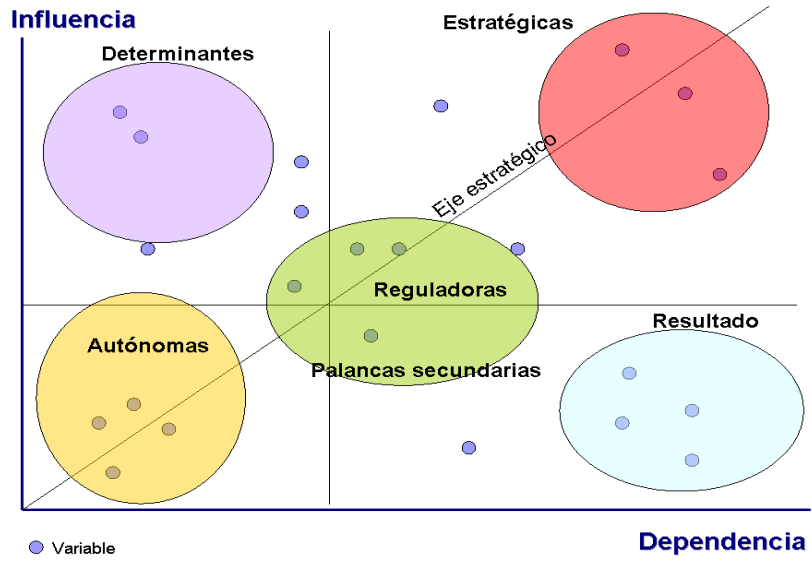

Fuente: elaboración propia con місмас 
El mapa resulta muy revelador, pues ilustra con gran acierto y detalle el funcionamiento del sistema. Para comenzar, merece la pena detenerse en las “variables autónomas", localizadas en el cuadrante inferior izquierdo. Dichas variables son poco influyentes $y$ dependientes, y pueden ser tendencias pasadas o, como en este caso, inercias sistémicas (Astigarraga, 2009). Para comenzar, en ese cuadrante inferior izquierdo se sitúa la variable "agua”, que hacía referencia a la escasez de agua de EE.UU. y a los planes estadounidenses para paliarla, incluyendo el IIRSA. Es acertado, entonces, categorizarla como inercia sistémica. Su dependencia respecto de las demás variables es nula, puesto que las acciones que se lleven a cabo en las otras en nada alterarán la escasez de agua de Ee.uu. Pero, además, el mapa revela cómo la influencia que ejerce sobre el sistema es muy escasa. Y es que concretamente el IIRSA parece, comparado con el Plan Mesoamérica o el NAWAPA, el menos viable para trasladar agua hasta EE.uU. Por ello los esfuerzos de este país respecto del proyecto no influirán en gran medida en el resto del sistema.

$\mathrm{Al}$ otro lado del cuadrante inferior izquierdo, justo con una dependencia media del sistema, pero con una influencia tan escasa como la anterior, se encuentra la variable "SAG", que corresponde a la evolución del Sistema Acuífero Guaraní. El por qué de su escasa influencia se asemeja al de la variable “agua”, ya que, como ocurre con el IIRSA, las opciones reales de EE.UU. de extraer agua del acuífero y trasportarla hacia el norte resultan complicadas en la actualidad, dadas las enormes dificultades técnicas y la larga distancia que los separan. No en vano, el miedo que se venía generando desde fuentes latinoamericanas apuntaba principalmente a la privatización de la gestión del acuífero, sobre todo a raíz del proyecto dirigido por el BM. Sin embargo, ni este proyecto ni el ALca han llegado a buen puerto, lo que reduce los riesgos en este sentido. Además, si finalmente Paraguay decidiera ratificar el Acuerdo multilateral firmado por los cuatro Estados que albergan el SAG, que parece más factible hoy tras normalizarse las relaciones en el seno del Mercosur, se habría dado un importante paso hacia el control del sag por parte de dichos Estados. No obstante, el que ciudadanos estadounidenses hayan adquirido terrenos sobre el SAG continúa siendo un elemento a tener en cuenta. Es indudable que, si bien la "expropiación" que según algunas fuentes pretendía realizar EE.uU. del agua del sAg no se ha producido, el camino más fácil para la eventual explotación estadounidense del mismo pasaría por la iniciativa privada. Por otro lado, todas estas vicisitudes explicarían por qué el sAg ocupa una posición de dependencia media en el sistema.

Otra variable "autónoma" destacable es “energía”, en referencia a la futura autosuficiencia energética de EE.UU. a partir de 2035 y que ya hoy ha comenzado a tener efectos sobre la economía del país. Como se ha dicho, este logro 
permitirá a EE.UU. relajar sus esfuerzos exteriores para asegurar los flujos energéticos mundiales, incluyendo los latinoamericanos. Esta autosuficiencia será prácticamente independiente de los cambios que se produzcan en el sistema que se está estudiando, al tiempo que influirá en ellos de manera relativa y casi exclusivamente sobre los Estados latinoamericanos exportadores de hidrocarburos. Para concluir con el cuadrante inferior izquierdo del mapa, en él también se sitúa la variable "China", que linda precisamente con el eje estratégico, lo que indica que su nivel de dependencia y su nivel de influencia son prácticamente iguales, aunque por tratarse de una variable "autónoma", este nivel es reducido. La creciente influencia e inversión de China en América Latina es una inercia sistémica que hasta ahora no ha sido enfrentada directamente por EE.UU., sobre todo teniendo en cuenta la limitada proyección estadounidense - ya mencionada - sobre dicha región durante la última década. En la actualidad, China es el principal reto geoestratégico de Washington, pero su ubicación en la parte inferior izquierda del mapa indica que la estrategia de EE.UU. consiste en emplear otros medios y en centrarse en otras regiones del mundo para hacer frente a su empuje.

En el extremo opuesto del mapa, cuadrante superior derecho, se ubican las "variables estratégicas", las más importantes del sistema, que por su elevada dependencia e influencia tienden a sobredeterminarlo. Las acciones que se ejerzan sobre ellas, así como aquellas ejercidas por cada una, afectarán de manera rotunda al conjunto del sistema (Astigarraga, 2009). Entre ellas se hallarán, por tanto, las variables que realmente dan sentido al mismo. Lo primero destacable de este cuadrante del mapa es la prominente posición del terrorismo dentro de él. La variable "terrorismo" es la que más influye sobre el resto, y se sitúa en el extremo más arriba y más a la derecha del cuadrante; pero en dicho cuadrante se ubican, además, los tres Estados que convergen en la тва, que se sitúan por debajo del terrorismo a lo largo del eje estratégico. Venezuela, considerada por muchos la nueva base de Hezbollah en América Latina, también se ubica en este cuadrante. Por tanto, cabría pensar que el interés y la presencia de EE.uU. en la tBA estarían motivados fundamentalmente por el terrorismo. No obstante, como hemos dicho, ello no significa que realmente existan células de Hezbollah, sino que la motivación de Washington para destinar esfuerzos a esa zona ha sido y es el terrorismo, por encima de otros como el agua, los hidrocarburos o China, que ocupan un lugar inferior en el mapa. Por tanto, será de la evolución del terrorismo, más que estos otros intereses, de lo que realmente dependan el interés y la presencia estadounidense en la тва.

La posición que ocupan los Estados que convergen en la тва — siempre a lo largo del eje estratégico- obedece 
a sus particulares características. Brasil goza de una posición prominente por ser potencia regional y por la presencia activa del terrorismo en su territorio más allá de Foz do Iguaçu, pues numerosas fuentes indican que la red de Rabbani se extiende hasta Brasil. Por tanto, las acciones de este Estado, en esta y otras materias, serán de vital importancia. Por otro lado, Ciudad del Este es la más conflictiva de las integrantes del triángulo transfronterizo, y donde mayor actividad de Hezbollah se ha registrado a lo largo del tiempo, sobre todo financiera. Además, Paraguay mantiene una posición de gran importancia debido a la presencia militar de EE.UU. en la base de Mariscal Estigarribia, cuya ubicación geográfica permite vigilar lo que ocurra en la TBA, y también la evolución de los hidrocarburos bolivianos y el devenir de las FARC en Colombia. Asimismo, la importancia de Argentina en el mapa es manifiesta, por ser el Estado que ha sufrido más directamente el terrorismo yihadista. El fiscal Nisman es un actor a considerar por sus esfuerzos en esclarecer los dos atentados. De hecho, recientemente ha acusado nuevamente a Hezbollah e Irán de estar detrás de los mismos. A estos tres Estados se une Venezuela que - como hemos indicado - en los últimos tiempos ha centrado la atención de numerosas fuentes que sitúan en Isla Margarita la base de Hezbollah en el continente. Además, durante la última década las relaciones entre Venezuela e Irán han sido muy intensas, lo que ha despertado recelo en EE.UU. por la difusión del sentimiento anti-estadounidense en América Latina que ello conllevaba. Así, habrá que tener muy en cuenta la evolución de la variable Venezuela, pues actualmente atraviesa una crisis que, de resolverse a favor de la oposición, podría beneficiar los intereses de Washington.

Por último, hay que destacar dentro las variables estratégicas, la "Geoestrategia”. Lógicamente el interés y la presencia de EE.uU. en la твA dependerán, en gran medida, de las decisiones de estrategia general que adopte la Casa Blanca. En la definición de las variables se ha visto cómo esa estrategia había pasado por alto, en buena parte, la evolución de América Latina durante la última década. De producirse un viraje en las líneas geoestratégicas generales, la presencia en la твА variaría en el mismo sentido. Pero no solo la "Geoestrategia" ejerce gran influencia sobre el sistema, sino que el mapa también revela que la evolución de la твA puede afectar a la geoestrategia global estadounidense.

Pasando ahora a las "variables reguladoras" y a las "palancas secundarias”, ubicadas en la mitad (superior e inferior, respectivamente) del mapa, ambas cumplen una función de "llave de paso", al determinar el funcionamiento del sistema y alterar el comportamiento de las "variables estratégicas” (Astigarraga, 2009). Debido a estas características, no sorprende encontrarnos en esta zona del mapa, dentro de la elipse verde, variables como 
Colombia o "narcotráfico". Ambas se encuentran relacionadas de manera evidente entre sí, así como ocurre con "terrorismo" o "Venezuela", tal y como se ha explicado. Además, las dos se ubican junto al eje estratégico, lo que hace aún más significativa su función. Parece claro, entonces, que el resultado de las negociaciones de paz entre el gobierno colombiano y las FARC afectará de forma decisiva al "narcotráfico", lo que generaría consecuencias directas en las variables superiores, como por ejemplo a aquellos dirigentes venezolanos presuntamente implicados en el tráfico de drogas, de acuerdo con las acusaciones vertidas desde EE.UU. También tendría consecuencias para Paraguay — ruta habitual del narcotráfico hacia Brasil, desde donde parte hacia EE.Uu. y Europa-, que lógicamente también se vería afectada, y específicamente pen el caso de la TBA, foco habitual del narcotráfico. Esta cadena causal afectaría finalmente al terrorismo, en la medida en que el narcotráfico sea medio de financiación del mismo. De ahí que la evolución del narcotráfico y del proceso de paz en Colombia es crucial para el sistema.

Mención aparte merece la presencia de la variable Irán justo en el centro del mapa, lo que indica que el Estado persa juega un papel clave, ya que su posición como patrocinador de $\mathrm{Hez}-$ bollah es algo generalmente aceptado. A ello se suma la acusación, en este sentido, del fiscal Nisman de 20I3. Por tanto, la evolución de la variable Irán es vital para el desarrollo del sistema, que - como hemos visto- queda definido por la presencia del terrorismo y concretamente de Hezbollah. Es aquí donde el desarrollo del acuerdo nuclear de fines de 2013 entre EE.uU. y el Estado persa cobra gran importancia. Este acuerdo supone un acercamiento que trata de normalizar las relaciones entre ambos y que podría traducirse en un aumento del peso relativo de Irán en Oriente Medio. No obstante, para que esto suceda, Washington exigirá ciertas garantías, una de las cuales podría ser la realización de un mayor control sobre la actividad terrorista de Hezbollah. En este sentido, se crearon previsiones bastante halagüeñas en Occidente cuando Rohani sustituyó a Ahmadineyad como Presidente de Irán, debido al carácter moderado del primero. No obstante, autores como Levitt aseguran, sobre la base de que el patrocinio iraní del terrorismo ha continuado estable o aumentado durante los mandatos de otros presidentes considerados como moderados, que la actividad de Hezbollah no se verá mermada durante el Gobierno Rohani (Levitt, 20I3: I0-II). Sea como fuere, por su posición central en el mapa, la evolución de Irán marcará, en gran medida, el devenir de la тва.

Para terminar, cabe destacar la posición marginal que ocupa la variable dependiente "interés". Como tal, ocupa lógicamente una posición de dependencia total en el margen derecho del mapa, pero su escasa influencia parece evidenciar que el interés y la presencia estadounidenses en la твA 
no son un factor determinante en el sistema y, por tanto, parecen limitadas las opciones de EE.uU. de modificar las tendencias de la тва, aumentando simplemente su presión sobre la misma.

\section{Conclusiones}

Este artículo ha tratado de presentar de manera ordenada los argumentos esgrimidos por las distintas partes, con el fin de arrojar luces sobre la presencia de terrorismo en la тва y sobre los intereses que EE.UU. podría tener en dicha zona. En este sentido, parece demostrado que la presencia de terrorismo yihadista está vinculada casi exclusivamente al grupo libanés Hezbollah, y que las actividades de este, cuando existan y al margen de los atentados de Buenos Aires de principios de los años ' 90 , serían de carácter fundamentalmente financiero. En cuanto al resto de intereses, la mayoría de fuentes latinoamericanas hacen referencia a los hidrocarburos, la influencia china, el narcotráfico y, sobre todo, el agua del SAG. Sobre esta última existía el temor de que la combinación del Proyecto de Sostenibilidad del sAG — financiado por el BM-y del ALCA supusiese la privatización y expropiación de este valiosísimo recurso natural.

Estos planteamientos han estado vigentes durante la mayor parte de la primera década del siglo xxi. No obstante, algunos acontecimientos a nivel internacional, que se han venido produciendo en los últimos años, también podrían haber afectado la situación en la твA y los intereses de EE.UU. en dicha zona. Este artículo, por tanto, ha tratado de combinar todos los datos, actores e intereses y, estudiando su evolución ha intentado comprobar si es cierto que el devenir de los acontecimientos internacionales ha podido disminuir la importancia de la твA, así como el interés y la presencia estadounidenses en ella. Así, los resultados de nuestro análisis nos ayudan a entender mejor la situación y nos habilitan para dar respuesta a esa cuestión. Como se ha visto, tres de los cuatro intereses que, según algunas fuentes, podría tener EE.UU. en la TBA, aparte del terrorismo, no han resultado ser tan determinantes como podía parecer. En efecto, ni la búsqueda de hidrocarburos, ni la influencia de China, ni siquiera el agua del sAG, son elementos que definan la presencia de EE.UU. en la твA, a tenor de la evolución que estas tres variables han experimentado. Esto no significa que dichos intereses no existan, sino que por sí mismos no explicarían la situación. Al ser inercias del sistema, como las hemos definido, han de ser tenidas en cuenta y es razonable pensar que Ee.uU. pudiera aprovechar su presencia en la TBA para abordarlas, pero otras estrategias más concretas habrán de ser puestas en marcha desde Washington para tratar, 
de forma concreta, cada una de estas tres cuestiones.

El otro interés que se atribuía a EE.UU. era el control de la situación en Colombia y del narcotráfico. Como se ha dicho, estas variables ejercen una influencia mucho más determinante que las anteriores por la conexión de la твA con el terrorismo y por la fuerte presencia del narcotráfico en la zona. No obstante, no llegan a ser variables estratégicas, y la presencia de planes concretos por parte de EE.UU. para combatir esta situación, como el Plan Colombia, parecen indicar que no constituye el fundamento principal del interés estadounidense. Al contrario, es la presencia de terrorismo, focalizado en Hezbollah, la que determina el sistema, el verdadero reto del mismo y cuya evolución marcará el interés y la presencia estadounidense en la твA de cara al futuro. Teniendo esto claro, y regresando a la cuestión que queremos resolver, parece que efectivamente los últimos acontecimientos podrían hacer decrecer ese interés por parte de
Washington, validándose así la hipótesis de este artículo. Y es que el traslado de las redes operativas de Hezbollah hacia Venezuela, así como el proceso de normalización de relaciones que puede tener lugar entre Irán y EE.UU., podrían ocasionar que la presencia del terrorismo en la тва, cualquiera que esta haya sido, disminuya. Si a ello sumamos que el resto de intereses "secundarios", que ya hemos explicado, han experimentado tendencias similares, hemos de confirmar nuestra hipótesis de que el interés y la presencia de EE.UU. en esta región han disminuido, y continuarán haciéndolo mientras los actuales factores se mantengan. No obstante, dadas las características de la TBA, esta zona siempre será objeto de preocupación tanto para los Estados que convergen en ella como para EE.uU., por lo que el interés sobre ella no desaparecerá por completo, sino que los actores se mantendrán alerta por si los acontecimientos volviesen a cambiar.

\section{Bibliografía}

ABC Color (20II). "Criminalidad se instala en Salto del Guairá”, II de mayo. Recuperado el 06 de noviembre de 2017 de http://abc.com.py/articulos/ criminalidad-se-instala-en---salto-delguaira-256318.html

ABC Color (2012). "Rechazan el protocolo para manejo de Acuífero Guaraní," 3 I de agosto. Recuperado el 06 de noviembre de 2017 de http://abc.com. py/edicion-impresa/politica/rechazan-el-protocolo-para-manejo-de-acuifero-guarani-444985.html

Agencia Internacional de la Energía (AIE) (20I3). World Energy Outlook 20I3. Resumen Ejecutivo. París: IEA Publications. 
AMERIPOL (20I3). Análisis situacional del narcotráfico. Una perspectiva policial. Madrid: FIIAP.

AMIA Case. Recuperado el 06 de noviembre de 2017 de http://defenddemocracy. org/stuff/uploads/documents/summary_(3I_pages).pdf

Anzit, R. (2006). La Triple Frontera (Brasil, Argentina y Paraguay): ¿Terrorismo o criminalidad? Recuperado el 06 de noviembre de 2017 de http://anzit-guerrero.net/admin/pdf/7I288403.pdf

Astigarraga, E. (2009). MIC MAC. Análisis Estructural. Recuperado el $\circ 6$ de noviembre de 2017 de http:// prospectiva.eu/curso-prospectiva/ programas_prospectiva/micmac

Bartolini, S. (I995), "Metodología de la investigación política”, en Pasquino, G. (ed.), Manual de Ciencia Política. Madrid: Alianza Universidad Textos, pp. 39-78.

Bartolomé, M. (2013). Terrorismo Internacional: Hezbollah ha vuelto a ser noticia. Recuperado el 06 de noviembre de $20 I 7$ de http:/npsglobal.org/esp/ component/content/article/I5I-analisis/I465-plantilla-articulos.html

Biron, C.L. (20I2). Water Conflicts Move Up on U.S. Security Agenda. Recuperado el 06 de noviembre de 2017 de http://ipsnews.net/20I2/05/water-conflicts-move-up-on-us-security-agenda/

Bruzzone, E.M.; García, J.L., y Ballester, H.P. (2006). La patraña del peligro terrorista en la Triple Frontera. Buenos Aires: Centro de Militares para la Democracia Argentina (CEMIDA).

Caro, I. (20I2). Conexiones latinoamericanas del islamismo radical. De Al Qaeda a la Triple Frontera. Santiago de Chile: RIL Editores.

Country Reports on Terrorism 2008. Recuperado el 06 de noviembre de 2017 de http://state.gov/documents/organization/I22599.pdf

Dinatale, M. (20I4). Giro del Gobierno: corta el diálogo con Irán y estrecha lazos con Israel. Recuperado el 06 de noviembre de 2017 de http://lanacion. com.ar/I664790-giro-del-gobierno-corta-el-dialogo-con-iran-y-estrecha-lazoscon-israel

Ellis, E. (2013). Are Big Chinese Energy Investments in Latin America a Concern? Recuperado el 06 de noviembre de 2017 de http://manzellareport.com/ index.php/world/78I-are-big-chineseenergy-investments-in-latin-americaa-concern

Fray Bentos, S.D. (2009). Fracasó el acuerdo sobre el Acuífero Guaraní. Recuperado el 06 de noviembre de 2017 de http://1r2I.com.uy/politica/388388-fracaso-acuerdo-sobre-acuifero-guarani

Friedman, G. (2013). Israel's New Strategic Position. Recuperado el 06 de noviembre de 2017 de http://stratfor.com/weekly/israels-new-strategic-position?utm_source=freelist-f\&utm_medium=email \&utm c a m paig n = 20I 3 I $203 \&$ u t m . term=Gweekly\&utm_content=readmore

Godet, M. (2000). "El análisis estructural”, en Godet, M.; Monti, R.; Meunier, F., y Roubelat, F. (eds.), La caja de herramientas de la prospectiva estratégica. Zarautz-Guipuzkoa: Prospektiker, pp 68-73. 
Gosman, E. (2006). Triple Frontera: Argentina, Brasil y Paraguay coordinan su espionaje. Recuperado el 06 de noviembre de 2017 de http://edant.clarin.com/ diario/2006/08/16/elpais/p-0060r.htm

Guerrero, A. (2006): "La prospectiva y la actitud prospectiva”, Módulo i: Metodología aplicada a las cuestiones de seguridad, del Programa de Doctorado Análisis y Estudios de Seguridad. Granada: Universidad de Granada, pp. I-I5.

Hudson, R. (2003). Terrorist and organized crime groups in the Tri-Border Area (твA) of South America. Washington D.C.: Federal Research Division, Library of Congress.

Lasserre, F. (2005). "Los locos proyectos para transferir el agua”. Le Monde Diplomatique (edición española), no II3, pp. I8-I9.

Levitt, Ma. (20I3). Examining the State Department's report on Iranian presence in the Western Hemisphere nineteen years after AMIA attack. [Testimony submitted to the House Committee on Foreign Affairs, Subcommittee on the Middle East and North Africa and Subcommittee on the Western Hemisphere]. Washington D.C.

Levitt, M. (2007). "Hezbollah Finances: Funding the Party of God", en Giraldo, J.K., y Trinkunas, H.A. (eds.), Terrorism Financing and State Responses. A Comparative Perspective. Stanford: Stanford University Press, pp. I34-I5I.

Martínez, M. (dir). (2004). Sed. Invasión gota a gota [Documental].

Morán, S. (20II). "Brasil, el gigante de Sudamérica con vocación mundial: Un proyecto inacabado", en Instituto Español de Estudios Estratégicos (IEEE)-Ministerio de Defensa, Las potencias emergentes hoy: Hacia un nuevo orden mundial. Madrid: IEEE, pp. I2I-I63.

Noriega, R.F. y Cárdenas, J.R. (20II). La creciente amenaza de Hezbollah en América Latina. Recuperado el 06 de noviembre de 2017 de http://aei.org/ article/foreign-and-defense-policy/regional/latin-america/la-creciente-amenaza-de-hezbollah-en-amrica-latina/

Noriega, R.F. (20I2). Hezbollah's strategic shift: a global terrorist threat. [Testimony before the United States House of Representatives, Committee on Foreign Affairs, Subcommittee on terrorism, non proliferation and trade]. Washington D.C.

Proyecto Sistema Acuífero Guaraní. Recuperado el 06 de noviembre de 2017 de http://sg-guarani.org/

Sánchez de Rojas, E. (20II), "Seguridad, geopolítica y geoestrategia. ¿Ha cambiado la geopolítica de Estados Unidos en Latinoamérica?", en Centro Superior de Estudios de la Defensa Nacional (CESEDen)-Ministerio de Defensa, El futuro de las relaciones Latinoamérica-Estados Unidos. Madrid: CESEDEN, pp. IO3-I42.

Segrelles, J.A. (2010). “Geopolítica actual del agua en el mundo" [versión electrónica] Revista Sociedad Latinoamericana, $\mathrm{Vol}$ 3, no.2. Recuperado el $\circ 6 \mathrm{de}$ noviembre de 2017 de http://sociedadlatinoamericana.bligoo.com/content/ view/IO2I402/Geopolitica-actual-delagua-en-el-mundo.html\#content-top Shaikhzadeh Vahdat, M.A. (20I3). Hezbollah y la Triple Frontera. Percepciones 
y divergencias en torno a la actividad terrorista. Recuperado el 06 de noviembre de $20 \mathrm{I} 7 \mathrm{de}$ http://defonline.com. ar/?p=22092\#_ftn4

Stratfor (20I4). Venezuela's President reaches out to the opposition. Recuperado el 06 de noviembre de 2017 de http://stratfor.com/sample/analysis/ venezuelas-president-reaches-out-opposition

Sustaining U.S. Global Leadership: Priorities for 2Ist Century Defense.
Recuperado el 06 de noviembre de 20I7 de http://defense.gov/news/defense_strategic_guidance.pdf Szmolka, I. y de Cueto, C. (20II). Objeto y método de la política comparada. Granada: Universidad de Granada.

Water 2025: Preventing Crises and Conflict in the West. Recuperado el $06 \mathrm{de}$ noviembre de 2017 de http://biodiversity.ca.gov/Meetings/archive/watero3/ water2025.pdf 